\title{
An Instrument to Measure Mental Health Professionals' Beliefs and Attitudes towards Service Users' Rights
}

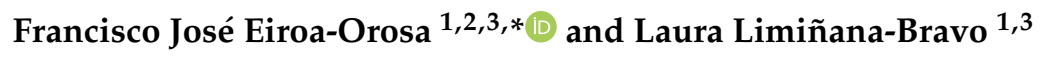 \\ 1 Section of Personality, Assessment and Psychological Treatment, Department of Clinical Psychology and \\ Psychobiology, Faculty of Psychology, University of Barcelona, Barcelona, 08035 Catalonia, Spain; \\ lauralb93@hotmail.com \\ 2 Yale Program for Recovery and Community Health, Department of Psychiatry, Yale School of Medicine, \\ Yale University, New Haven, CT 06513, USA \\ 3 First-Person Research Group, Veus, Catalan Federation of 1st Person Mental Health Organisations, \\ Barcelona, 08025 Catalonia, Spain \\ * Correspondence: fjeiroa@gmail.com or feiroa@ub.edu
}

Received: 26 November 2018; Accepted: 11 January 2019; Published: 16 January 2019

\begin{abstract}
We aimed at developing and validating a scale on the beliefs and attitudes of mental health professionals towards services users' rights in order to provide a valid evaluation instrument for training activities with heterogeneous mental health professional groups. Items were extracted from a review of previous instruments, as well as from several focus groups which have been conducted with different mental health stakeholders, including mental health service users. The preliminary scale consisted of 44 items and was administered to 480 mental health professionals. After eliminating non-discriminant and low weighting items, a final scale of 25 items was obtained. Exploratory and confirmatory factor analyses produced a four-factor solution consisting of the following four dimensions; system criticism/justifying beliefs, freedom/coercion, empowerment/paternalism, and tolerance/discrimination. The scale shows high concordance with our theoretical model as well as adequate parameters of explained variance, model fit, and internal reliability. Additional work is required to assess the cultural equivalence and psychometrics of this tool in other settings and populations, including health students.
\end{abstract}

Keywords: mental health services; attitudes; beliefs; coercion; paternalism; discrimination

\section{Introduction}

The mental health sector has undergone two fundamental transformations in the last half century, namely Deinstitutionalization and Recovery [1]. Both processes have involved an increase in service users' autonomy and freedom of choice. The Recovery movement has mostly been driven by service users themselves, which has entailed a significant increase in participation, and consequently a reduction in paternalistic behaviours carried out by professionals. These processes have also improved the professionals' awareness of service users rights, and have led to a reduction of coercive measures and a shift from symptom reduction to rehabilitative and recovery approaches [2].

Despite all these improvements, many service users still report stigmatizing attitudes, including professional paternalism and emotional estrangement $[3,4]$. Therefore, receiving a mental health diagnosis is still considered as a predisposing factor that can lead to the experience of stigma from both the social environment [5] and mental health professionals [6].

Stereotypes depicting mental health service users as incompetent, weak, incurable, and violent lead to social discrimination and coercive professional practices [7]. Some examples are involuntary 
inpatient and outpatient treatments, forced medication, overmedication, electroconvulsive therapy under duress, mechanical restraints, seclusion, isolation, and arbitrary legal incapacitations and guardianships. The underlying beliefs that influence the decisions that professionals make appear to be formed by a lack of awareness of one's own prejudices [4]. In this sense, the perception by some professionals that the moral side of their decisions is not relevant to the recovery process may contribute to the acceptance of coercion as a standard practice [8].

Given the extent of the consequences of stigma and coercion towards mental health service users, it is essential to raise awareness among mental health professionals in order to foster non-stigmatizing and empowering attitudes through frameworks such as Recovery [9] and Citizenship [10]. In the context of planning and implementation of these training and awareness activities, there is a need to evaluate the impact this has on the beliefs and attitudes of professionals through standardised measures.

\section{Previous Measures}

The first scale that included beliefs and attitudes among mental health professionals towards mental health service users was developed by Gilbert and Levinson [11]. This scale was used as a method to understand mental health practices on a continuum from custodial to humanistic [12]. Another scale dealing with professionals opinions about mental illness was validated shortly afterwards [13]. Alongside these early developments, Goffman re-defined the word "stigma" to refer to a non-physical, invisible signal, making a person's social status undesirable [14]. Relatedly, different mental health stigma measures have been developed and are usually applied to the general public [15-22], mental health service users [23], and some others to professional audiences [24-29]. Another issue that has also generated assessment tools is the stigma perceived by those in the medical profession in general towards psychiatry in particular [30].

Over the last decades, in parallel to the rise of the Rehabilitation and Recovery movements, professionals have become more conscious of the need to offer a non-discriminant care based on users' rights. In this context, measures on recovery-based knowledge [31-33], attitudes [34], expectations [35], and practices [36-42] have been developed (see Table 1). These instruments have enabled the evaluation of dozens of projects which have implemented the philosophy of recovery in many health institutions. However, there are also limitations of these instruments, including the impossibility of administering the attitudes and knowledge measures at baseline with lay professionals, given that they assume a certain degree of knowledge of the principles of recovery. Furthermore, all current professional stigma instruments are designed for certain professional groups [24-26,29] or mental health conditions $[27,28]$. Finally, scales measuring recovery practices assume that some level of implementation of these practices has already been done.

So far, there is a lack of multidimensional measures that can be used to assess training and awareness activities attended by different mental health professional groups with heterogeneous levels of knowledge and awareness of the importance of respecting service users' rights. For this reason, the objective of this work is the development of a flexible instrument in order to measure the beliefs and attitudes related to service users' rights among all types of mental health professionals.

\section{Materials and Methods}

\subsection{Development}

An initial set of 44 items was developed in Spanish by researchers with a lived experience of mental health problems. This set was reviewed by Catalan experts on stigma awareness and community mental health including board members of the Catalan Federation of First-Person Mental Health Organisations, where all the members have lived experience of mental health problems. Half of these items were derived from a systematic review of previous measures (see Table 1). We reviewed scales on stigmatizing attitudes and beliefs as well as recovery-based knowledge and practices. We found items 
related to three cognitive levels: attitudes, awareness and knowledge, and four thematic domains: empowerment/paternalism, recovery, stigma, and rights.

Additionally, we conducted 11 focus groups with mental health professionals, 7 focus groups with service users, and 1 focus group with relatives of service users. The first six groups carried with mental health professionals and those carried with service users and relatives were used for the adaptation to the current context of items based on the literature and the creation of a pool of 22 completely new items. In the last five groups carried with mental health professionals, the scale was presented at the beginning of the session, leaving time for participants to respond. During the discussion, professionals could comment on the content and contextualise how they had answered the items.

The order of the items was randomised before starting the administration of the scale. Regarding the anchor points of the scale, we chose a four-point Likert scale (I fully disagree, I disagree, I agree, I fully agree). This mode helps to minimise middle response bias, which is frequent in attitude research [43].

\subsection{Sample and Procedure}

The sample used for the psychometric validation of the Beliefs and Attitudes towards Mental Health Service Users' Rights Scale (BAMHS) was comprised of a total of 480 Spanish-speaking mental health professionals. These professionals worked in a diverse range of settings, including inpatient care, outpatient care, rehabilitation, supported work, leisure and free time services, etc. Among these, there were psychologists $(29 \%)$, mental health nurses $(15 \%)$, social educators working in mental health settings $(15 \%)$, psychiatrists $(12 \%)$, mental health social workers $(7 \%)$, occupational therapists $(4 \%)$, and other allied professionals including primary care doctors and nurses and non-specialists working with mental health service users such as community health workers and administrative staff $(15 \%)$. The average age was 40.13, ranging from 23 to 65 years of age. Approximately $77 \%$ of the sample were women.

Participants in the study were gathered from a pool of professionals who had participated in discussions, training sessions and awareness activities on mental health service user's rights. The scale was designed to be used as a baseline and follow-up measure.

The study received ethical clearance from the University of Barcelona institutional review board (IRB00003099). All participants gave informed consent and the questionnaires were completed anonymously.

\subsection{Analysis}

Before analysing the data, we carried out a search for outliers by calculating the mean of all the responses for each participant. We excluded a total of seven questionnaires from the analysis as they were indicative of extreme values (most answers corresponding to one of the Likert scale anchor points).

We calculated frequencies, asymmetry, and kurtosis parameters, as well as item-total correlations for each item in order to decide upon their inclusion in exploratory and confirmatory factor analyses (EFAs and CFAs, respectively), in addition to univariate and multivariate Item Response Theory (IRT) exploratory and confirmatory analyses. EFAs were combined with CFAs through the identification of stable and theoretically congruent dimensions appearing in consecutive principal components analyses, for which fit could be tested through structural equation modelling. In parallel, the discriminative capacity of each item was tested using uni- and multivariate IRT analyses. Through the analysis of item-total correlations, EFA, and CFA factor loading valences, items that indicated that they should be reversed were recoded, with higher scores indicating larger presence of negative attitudes or beliefs (see italicised items in Table S1). Finally, reliability was tested using Cronbach's alpha. The psych [44], lavaan [45], ltm [46], and mirt [47] packages for the R software [48] were used to compute all the statistical analyses. 
Table 1. Previous measures of mental health professionals' beliefs and attitudes towards service users' rights.

\begin{tabular}{|c|c|c|c|}
\hline Measure & Applies to & Constructs Measured & Reference \\
\hline Custodial Mental Illness Ideology Scale & Mental health professionals & Custodial and humanistic ideologies & {$[11,12]$} \\
\hline Opinions about Mental Illness Scale & Mental health professionals & Public stigma & [13] \\
\hline Community Attitudes towards the Mentally Ill Scale & General public & Public stigma & [16] \\
\hline Attitudes towards Psychiatry (ATP-30) & Mental health professionals & Attitudes towards the psychiatry specialty & [30] \\
\hline Perceived Devaluation and Discrimination Scale & General public/mental health service users & Public stigma & [20] \\
\hline Affective Reaction Scale & General public & Public stigma & {$[18,49]$} \\
\hline Dangerousness Scale & General public & Public stigma & {$[18,49]$} \\
\hline "Changing Minds" questionnaire & General public & Public stigma & [21] \\
\hline Recovery Attitudes Questionnaire (RAQ-7) & Mental health professionals & Recovery attitudes & [34] \\
\hline Professionals' Beliefs, Goals and Practices in Psychiatric Rehabilitation & Mental health professionals & Recovery practice & [36] \\
\hline Medical Condition Regard Scale & Medical students & Professional stigma & [27] \\
\hline Attribution Questionnaire & General public & Public stigma & [15] \\
\hline Internalized Stigma of Mental Illness (ISMI) & Mental health service users & Public stigma & [23] \\
\hline Recovery-Oriented Practices Index (ROPI) & Mental health professionals & Recovery practice & [41] \\
\hline Recovery Self-Assessment & $\begin{array}{l}\text { Mental health institutions (professionals, service } \\
\text { users and relatives) }\end{array}$ & Recovery practice & {$[42,50-52]$} \\
\hline Recovery Knowledge Inventory (RKI) & Mental health professionals & Recovery knowledge & [31] \\
\hline \multirow[t]{2}{*}{ Implicit Stigma (Implicit Association Test) } & General public & Public/professional stigma & [22] \\
\hline & Mental health professionals & & {$[25,40]$} \\
\hline Integrated Dual Disorders Treatment Model Knowledge Scale & Mental health professionals & Recovery knowledge & [33] \\
\hline $\begin{array}{l}\text { The Project GREAT Recovery Knowledge Measure-Recovery Attitudinal } \\
\text { Pre-Post Survey }\end{array}$ & Mental health professionals & Recovery knowledge & [32] \\
\hline Mental Health Knowledge Schedule & General public & Public stigma & [19] \\
\hline Mental Illness: Clinicians' Attitudes Scale (MICA) & Health professionals & Professional stigma & {$[26,53]$} \\
\hline Police Contact Experience Scale & Police officers & Professional stigma & [29] \\
\hline Reported and Intended Behaviour Scale (RIBS) & General public & & [17] \\
\hline Quality Indicator for Rehabilitative Care (QuIRC) & Mental health professionals & Recovery practice & [38] \\
\hline Opening Minds Stigma Scale for Health Care Providers (OMS-HC) & Primary care professionals & Professional stigma & [24] \\
\hline Consumer Optimism Scale & Mental health professionals & Professional optimism & [33] \\
\hline Provider Expectations for Recovery Scale & Mental health professionals & Professional optimism for recovery & {$[35]$} \\
\hline $\begin{array}{l}\text { PAREM (Attitude Questionnaire developed by Psychiatric Investigations and } \\
\text { Education Center) }\end{array}$ & Mental health students & Professional stigma & [28] \\
\hline Strengths Model Attitudes Questionnaire (SMAQ) & Mental health professionals & Recovery practice & [37] \\
\hline
\end{tabular}




\section{Results}

Frequencies, asymmetry, and kurtosis parameters for each original item can be seen in Table S1. Due to their low discriminative capacity, we decided to remove items with an asymmetry and kurtosis greater than 1 or less than -1 (eight items) and/or $90 \%$ of the cases included in one of the two halves of the Likert scale (nine additional items). We then calculated item-total correlations as well as a unidimensional IRT unconstrained latent variable model with the remaining 27 items. An item on professional pessimism (9), was removed because of nil $(r=-0.034)$ correlation with the rest of items and low discrimination parameter $(-0.057)$. All the remaining items had discrimination parameters above 0.5 within a unidimensional IRT model.

Consecutive exploratory factor analyses using Varimax and Oblimin rotations as well as exploratory IRT models were conducted with the 26 remaining items, using the eigenvalue-higher-than-one criterion in CFA and forcing the structure to 2, 3, and 4 factors in CFA and exploratory IRT. This procedure was repeated, temporarily excluding items with low and distributed loadings and low multivariate discriminant parameters. Once we identified a coherent item group (those that tended to remain under the same dimension with high factorial weights and discriminant parameters in different CFA and IRT analyses), we calculated its unidimensionality through Cronbach's alpha and confirmatory factorial analyses as well as the discrimination parameters of the items. Each group was subsequently removed from the total pool of items and the whole process was repeated with the remaining items, until we obtained a congruent model (19 items) formed by four dimensions.

The dimensions were named as follows: system criticism/justifying beliefs (items 3, 10, 15, 16, 44), freedom/coercion (items 4, 6, 23, 34), empowerment/paternalism (items 1, 8, 27, 28, 38, 40), and tolerance/discrimination (items $12,24,25,30$ ). The dimensionality of the core model was analysed through confirmatory factor analysis showing a good fit (see Table S2). Discrimination parameters within a multidimensional IRT (MIRT) were also satisfactory (0.80-2.62). Additional items were added one by one, based on theoretical coherence, factor loadings and discriminant parameters in all EFAs and confirmatory IRT models which were incorporated during the previous process (see Table S2). We again tested the EFA weights (Table 2), reliability (Table 3), and unidimensionality (Table S2) for the whole set of 25 items and for each dimension. We also tested the fit of the whole model having added only a specific item (see Table 3 and Table S2). All items added to each subscale (11, 13, 14, 33, 37, and 39) improved its internal reliability and the fit of the whole model considered as unidimensional, without substantially affecting the fit of the four-dimensional model. As it can be seen in Table S2, adding item 21 worsened all unidimensionality parameters. Additionally, we considered that it could be included as part of the tolerance/discrimination subscale. However, it did not improve the reliability of that subscale, nor any of the rest, and hence, it was removed.

Figure 1 shows the CFA path diagram of the final model. Discrimination parameters within the final MIRT were also satisfactory (0.70-2.04, see Table 2). The final scale can be seen in Table ?? (original version in Spanish) and Table 5 (back-translated version in English). 
Table 2. Factorial weights and discrimination data of the final item pool.

\begin{tabular}{|c|c|c|c|c|c|c|c|c|c|}
\hline \multirow[t]{2}{*}{$\begin{array}{c}\text { Original Item } \\
\text { Number }\end{array}$} & \multirow[t]{2}{*}{$\begin{array}{l}\text { Final Item } \\
\text { Number }\end{array}$} & \multirow[t]{2}{*}{ Statement } & \multicolumn{7}{|c|}{ EFA Weights * } \\
\hline & & & $\begin{array}{l}\text { System } \\
\text { criticism/ } \\
\text { justifying beliefs }\end{array}$ & $\begin{array}{l}\text { Freedom/ } \\
\text { coercion }\end{array}$ & $\begin{array}{l}\text { Empowerment/ } \\
\text { paternalism }\end{array}$ & $\begin{array}{l}\text { Tolerance/ } \\
\text { discrimination }\end{array}$ & $\begin{array}{l}\text { Univariate IRT } \\
\text { discriminant } \\
\text { parameter } \\
\text { within the } \\
\text { whole scale }\end{array}$ & $\begin{array}{l}\text { Univariate IRT } \\
\text { discriminant } \\
\text { parameter } \\
\text { within the } \\
\text { subscale }\end{array}$ & $\begin{array}{l}\text { Multivariate } \\
\text { IRT } \\
\text { discriminan } \\
\text { parameter }\end{array}$ \\
\hline & & System criticism/justifying beliefs & & & & & & & \\
\hline 3 & 2 & $\begin{array}{l}\text { It is possible to recover without professional } \\
\text { interventions. }\end{array}$ & 0.292 & & & & 1.229 & 1.091 & 1.088 \\
\hline 10 & 6 & Mental disorders are diseases like any other. & $0.257^{*}$ & & & & 0.639 & 0.853 & 0.856 \\
\hline 13 & 9 & $\begin{array}{l}\text { When patients behave aggressively it is due to their } \\
\text { mental disorder. }\end{array}$ & 0.639 & & & & 0.867 & 0.845 & 0.832 \\
\hline 14 & 10 & $\begin{array}{l}\text { Declaring someone with a severe mental disorder } \\
\text { incapacitated is a good way of taking care of that } \\
\text { person. }\end{array}$ & 0.471 & & & & 1.867 & 1.444 & 1.422 \\
\hline 15 & 11 & $\begin{array}{l}\text { Individuals with mental disorders currently have } \\
\text { the same rights as other people. }\end{array}$ & 0.463 & & & & 0.754 & 0.986 & 0.991 \\
\hline 16 & 12 & $\begin{array}{l}\text { Coercive measures are currently applied only when } \\
\text { necessary. }\end{array}$ & 0.552 & & & & 1.929 & 2.630 & 2.677 \\
\hline 39 & 23 & Some patients will never be able to recover. & $0.210^{*}$ & & & & 1.332 & 0.955 & 0.945 \\
\hline \multirow[t]{2}{*}{44} & 25 & $\begin{array}{l}\text { For the most part, mental health professionals work } \\
\text { collaboratively with patients. }\end{array}$ & 0.504 & & & & 1.098 & 1.465 & 1.484 \\
\hline & & Freedom/coercion & & & & & & & \\
\hline 4 & 3 & $\begin{array}{l}\text { People should not be involuntarily hospitalised if } \\
\text { they do not pose a threat to the integrity of others }\end{array}$ & & 0.638 & & & 1.066 & 1.584 & 1.644 \\
\hline 6 & 4 & $\begin{array}{l}\text { Sometimes it is necessary to mechanically restrain } \\
\text { patients. }\end{array}$ & & 0.557 & & & 1.367 & 1.967 & 1.907 \\
\hline 23 & 13 & $\begin{array}{l}\text { When a patient behaves aggressively it is due to the } \\
\text { situations, that occur for example in involuntary } \\
\text { admissions. }\end{array}$ & & 0.757 & & & .577 & 1.011 & 1.016 \\
\hline 34 & 20 & $\begin{array}{l}\text { Greater importance should be placed on promoting } \\
\text { the patient's independence than on reducing the } \\
\text { patient's symptoms. }\end{array}$ & & 0.367 & & & 1.264 & 1.166 & 1.187 \\
\hline \multirow[t]{2}{*}{37} & 21 & $\begin{array}{l}\text { If there are not enough staff, mechanical restraints } \\
\text { are the only way to manage violent situations. }\end{array}$ & & $0.137^{*}$ & & & 1.058 & 0.898 & 0.892 \\
\hline & & Empowerment/paternalism & & & & & & & \\
\hline 1 & 1 & $\begin{array}{l}\text { The possibility of people with severe mental } \\
\text { disorders having children should be regulated. }\end{array}$ & & & 0.496 & & 1.208 & 1.311 & 1.318 \\
\hline 8 & 5 & $\begin{array}{l}\text { Patients with severe mental disorders require } \\
\text { clearer instructions than other patients. }\end{array}$ & & & 0.359 & & 1.254 & 1.339 & 1.346 \\
\hline
\end{tabular}


Table 2. Cont

\begin{tabular}{|c|c|c|c|c|c|c|c|}
\hline 11 & 7 & $\begin{array}{l}\text { Professionals should have more say than patients in } \\
\text { making treatment decisions. }\end{array}$ & $0.115^{*}$ & & 1.969 & 1.536 & 1.585 \\
\hline 27 & 16 & $\begin{array}{l}\text { People with severe mental disorders always require } \\
\text { support to be able to live independently. }\end{array}$ & 0.416 & & 1.408 & 1.494 & 1.500 \\
\hline 28 & 17 & $\begin{array}{l}\text { Objective tests should be prioritised over the } \\
\text { professionals' and patients' opinion. }\end{array}$ & 0.591 & & 0.632 & 0.698 & 0.699 \\
\hline 33 & 19 & $\begin{array}{l}\text { Respecting the patients' dignity is important, but } \\
\text { some aspects of treatment may require flexibility. }\end{array}$ & 0.240 & & 1.022 & 1.016 & 1.032 \\
\hline 38 & 22 & $\begin{array}{l}\text { When dealing with patients it is important for me } \\
\text { not to get emotionally involved. }\end{array}$ & 0.617 & & 1.307 & 1.482 & 1.472 \\
\hline \multirow[t]{2}{*}{40} & 24 & $\begin{array}{l}\text { In my clinical practice I try to leave my personal } \\
\text { values aside. }\end{array}$ & 0.538 & & 0.812 & 0.877 & 0.888 \\
\hline & & Tolerance/discrimination & & & & & \\
\hline 12 & 8 & $\begin{array}{l}\text { Individuals incapacitated by severe mental health } \\
\text { problems should have the right to vote. }\end{array}$ & & 0.474 & 1.845 & 1.541 & 1.476 \\
\hline 24 & 14 & $\begin{array}{l}\text { I would feel comfortable making friends with } \\
\text { someone with a severe mental disorder. }\end{array}$ & & 0.534 & 1.148 & 1.786 & 1.827 \\
\hline 25 & 15 & $\begin{array}{l}\text { I am uncomfortable with patients who regularly } \\
\text { use emergency services. }\end{array}$ & & 0.634 & 0.850 & 1.121 & 1.150 \\
\hline 30 & 18 & $\begin{array}{l}\text { I would be comfortable if a person with a mental } \\
\text { disorder were a teacher in a school. }\end{array}$ & & 0.602 & 1.443 & 1.954 & 2.035 \\
\hline
\end{tabular}

* Items marked with an asterisk were the only four that weighted higher within a different dimension when performing exploratory factor analysis (EFA) with the final item set. IRT: Item Response Theory; EFA: exploratory factor analysis. 


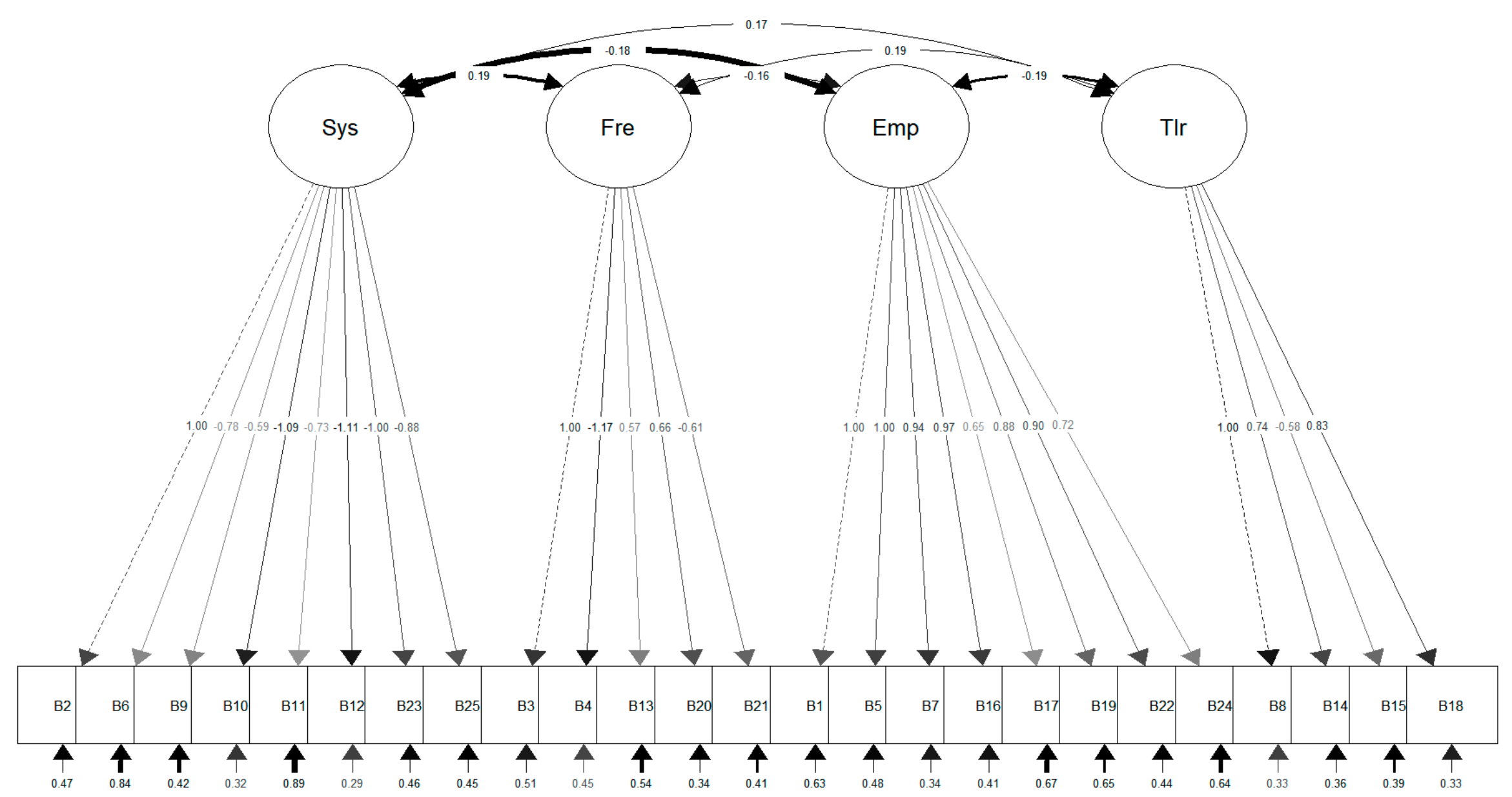

Figure 1. Structural equations diagram of the scale. 
Table 3. Reliability of the core structure, total scale, and four final dimensions including additional items *.

\begin{tabular}{|c|c|}
\hline Dimensions & Cronbach's Alpha \\
\hline Core structure (19 items) & 0.824 \\
\hline Total structure (25 items) & 0.867 \\
\hline \multicolumn{2}{|l|}{ Dimension 1-System criticism/justifying beliefs } \\
\hline $\begin{array}{l}\text { Core ( } 5 \text { items: } 3 / 2 \text { It is possible to recover without professional interventions, } 10 / 6 \\
\text { Mental disorders are diseases like any other, } 15 / 11 \text { Individuals with mental } \\
\text { disorders currently have the same rights as other people, } 16 / 12 \text { Coercive measures } \\
\text { are currently applied only when necessary, } 44 / 25 \text { For the most part, mental health } \\
\text { professionals work collaboratively with patients) }\end{array}$ & 0.644 \\
\hline Core $+13 / 9$ (When patients behave aggressively it is due to their mental disorder). & 0.644 \\
\hline $\begin{array}{l}\text { Core }+14 / 10 \text { (Declaring someone with a severe mental disorder incapacitated is a } \\
\text { good way of taking care of that person). }\end{array}$ & 0.681 \\
\hline Core $+39 / 23$ (Some patients will never be able to recover) & 0.649 \\
\hline Final (8 items) & 0.706 \\
\hline \multicolumn{2}{|l|}{ Dimension 2-Freedom/coercion } \\
\hline $\begin{array}{l}\text { Core ( } 4 \text { items: } 4 / 3 \text { People should not be involuntarily hospitalised if they do not } \\
\text { pose a threat to the integrity of others, } 6 / 4 \text { Sometimes it is necessary to } \\
\text { mechanically restrain patients, } 23 / 13 \text { When a patient behaves aggressively it is } \\
\text { due to the situations that occur, for example in involuntary admissions, } 34 / 20 \\
\text { Greater importance should be placed on promoting the patient's independence } \\
\text { than on reducing the patient's symptoms). }\end{array}$ & 0.641 \\
\hline $\begin{array}{l}\text { Core }+37 / 21 \text { (If there are not enough staff, mechanical restraints are the only way } \\
\text { to manage violent situations). }\end{array}$ & 0.652 \\
\hline \multicolumn{2}{|l|}{ Dimension 3-Empowerment/paternalism } \\
\hline $\begin{array}{l}\text { Core ( } 6 \text { items: } 1 / 1 \text { The possibility of people with severe mental disorders having } \\
\text { children should be regulated, } 8 / 5 \text { Patients with severe mental disorders require } \\
\text { clearer instructions than other patients, } 27 / 16 \text { People with severe mental disorders } \\
\text { always require support to be able to live independently, } 28 / 17 \text { Objective tests } \\
\text { should be prioritised over the professionals' and patients' opinion, } 38 / 22 \text { When } \\
\text { dealing with patients it is important for me not to get emotionally involved, } 40 / 24 \\
\text { In my clinical practice I try to leave my personal values aside). }\end{array}$ & 0.658 \\
\hline $\begin{array}{l}\text { Core }+11 / 7 \text { (Professionals should have more say than patients in making } \\
\text { treatment decisions). }\end{array}$ & 0.689 \\
\hline $\begin{array}{l}\text { Core }+33 / 19 \text { (Respecting the patients' dignity is important, but some aspects of } \\
\text { treatment may require flexibility). }\end{array}$ & 0.676 \\
\hline Final (8 items) & 0.709 \\
\hline \multicolumn{2}{|l|}{ Dimension 4-Tolerance/discrimination } \\
\hline $\begin{array}{l}\text { Core/Final ( } 4 \text { items: } 12 / 8 \text { Individuals incapacitated by severe mental health } \\
\text { problems should have the right to vote, } 24 / 14 \text { I would feel comfortable making } \\
\text { friends with someone with a severe mental disorder, } 25 / 15 \text { I am uncomfortable } \\
\text { with patients who regularly use emergency services, } 30 / 18 \text { I would be comfortable } \\
\text { if a person with a mental disorder were a teacher in a school). }\end{array}$ & 0.650 \\
\hline
\end{tabular}


Table 4. Spanish Version of the Beliefs and Attitudes towards Mental Health Service Users' Rights Scale including scoring details.

\begin{tabular}{|c|c|c|c|c|}
\hline $\begin{array}{l}\text { En relación a pacientes con problemas de salud mental y como } \\
\text { profesional pienso que ... }\end{array}$ & $\begin{array}{l}1 \text { Totalmente en } \\
\text { desacuerdo }\end{array}$ & $\begin{array}{l}2 \text { En } \\
\text { desacuerdo }\end{array}$ & $\begin{array}{l}3 \text { De } \\
\text { acuerdo }\end{array}$ & $\begin{array}{l}4 \text { Totalmente de } \\
\text { acuerdo }\end{array}$ \\
\hline $\begin{array}{l}\text { 1. Se debería regular la posibilidad de que las personas } \\
\text { con trastornos mentales severos tengan hijos. }\end{array}$ & $\square$ & $\square$ & $\square$ & $\square$ \\
\hline $\begin{array}{ll}\text { 2. } & \text { Es posible recuperarse sin la intervención de un } \\
\text { profesional. }\end{array}$ & $\square$ & 口 & 口 & $\square$ \\
\hline $\begin{array}{l}\text { 3. No se debería hospitalizar involuntariamente si no } \\
\text { hay peligro para la integridad de terceras personas. }\end{array}$ & $\square$ & 口 & $\square$ & $\square$ \\
\hline 4. Las contenciones mecánicas a veces son necesarias. & ㅁ & ㅁ & 口 & ㅁ \\
\hline $\begin{array}{l}\text { 5. Los pacientes con trastorno mental severo necesitan } \\
\text { pautas más claras que el resto. }\end{array}$ & $\square$ & 口 & 口 & 口 \\
\hline $\begin{array}{l}\text { 6. Los trastornos mentales son enfermedades como } \\
\text { cualquier otra. }\end{array}$ & $\square$ & 口 & 口 & 口 \\
\hline $\begin{array}{l}\text { 7. Los profesionales debemos tener mayor prioridad } \\
\text { que los pacientes para tomar decisiones sobre su } \\
\text { tratamiento. }\end{array}$ & 口 & 口 & 口 & 口 \\
\hline $\begin{array}{ll}\text { 8. } & \text { Las personas incapacitadas por problemas de salud } \\
\text { mental severos deberían tener derecho a votar. }\end{array}$ & $\square$ & 口 & 口 & $\square$ \\
\hline $\begin{array}{l}\text { 9. Cuando un paciente lleva a cabo una agresión es por } \\
\text { causa de su trastorno mental. }\end{array}$ & $\square$ & 口 & 口 & $\square$ \\
\hline $\begin{array}{l}\text { 10. Una incapacitación es una buena manera de hacerse } \\
\text { cargo de una persona con un trastorno mental } \\
\text { severo. }\end{array}$ & $\square$ & 口 & 口 & 口 \\
\hline $\begin{array}{l}\text { 11. Las personas con trastorno mental tienen hoy en día } \\
\text { los mismos derechos que el resto de personas. }\end{array}$ & $\square$ & $\square$ & $\square$ & $\square$ \\
\hline $\begin{array}{l}\text { 12. Actualmente se aplican medidas coercitivas sólo } \\
\text { cuando es necesario. }\end{array}$ & 口 & 口 & $\square$ & $\square$ \\
\hline $\begin{array}{l}\text { 13. Cuando un paciente lleva a cabo una agresión es por } \\
\text { causa de las situaciones que se dan por ejemplo en } \\
\text { los ingresos involuntarios. }\end{array}$ & 口 & $\square$ & 口 & 口 \\
\hline $\begin{array}{l}\text { 14. Me sentiría cómodo haciéndome amigo de alguien } \\
\text { con un trastorno mental severo. }\end{array}$ & 口 & $\square$ & 口 & 口 \\
\hline $\begin{array}{l}\text { 15. Me incomodan los pacientes que frecuentan los } \\
\text { servicios de urgencias. }\end{array}$ & 口 & 口 & 口 & 口 \\
\hline $\begin{array}{l}\text { 16. Las personas con trastorno mental severo siempre } \\
\text { necesitan apoyo para poder hacer vida autónoma. }\end{array}$ & $\square$ & $\square$ & 口 & 口 \\
\hline $\begin{array}{l}\text { 17. Deberían priorizarse pruebas objetivas sobre las } \\
\text { opiniones de profesional y paciente en el } \\
\text { planteamiento de un caso. }\end{array}$ & 口 & $\square$ & $\square$ & $\square$ \\
\hline $\begin{array}{l}\text { 18. Me sentiría cómodo si una persona con trastorno } \\
\text { mental fuera profesor en una escuela. }\end{array}$ & $\square$ & $\square$ & $\square$ & 口 \\
\hline $\begin{array}{l}\text { 19. El respeto a la dignidad de los pacientes es } \\
\text { importante, pero a veces el tratamiento exige ser } \\
\text { flexible con algunos aspectos. }\end{array}$ & $\square$ & 口 & 口 & $\square$ \\
\hline $\begin{array}{l}\text { 20. Se debería priorizar la autonomía de los pacientes } \\
\text { por encima de la disminución de síntomas. }\end{array}$ & $\square$ & 口 & 口 & 口 \\
\hline $\begin{array}{l}\text { 21. Si no hay personal suficiente, las contenciones } \\
\text { mecánicas son la única manera de poder gestionar } \\
\text { situaciones violentas. }\end{array}$ & $\square$ & 口 & 口 & 口 \\
\hline $\begin{array}{l}\text { 22. En el trato con los pacientes para mi es importante } \\
\text { no implicarme emocionalmente. }\end{array}$ & 口 & $\square$ & 口 & 口 \\
\hline 23. Algunos pacientes no podrán recuperarse nunca. & $\square$ & $\square$ & $\square$ & $\square$ \\
\hline $\begin{array}{l}\text { 24. En mi práctica clínica intento dejar mis valores } \\
\text { personales de lado. }\end{array}$ & 口 & 口 & 口 & 口 \\
\hline $\begin{array}{l}\text { 25. La mayor parte de los profesionales de salud mental } \\
\text { trabajamos de un modo colaborativo con los } \\
\text { pacientes. }\end{array}$ & $\square$ & $\square$ & $\square$ & $\square$ \\
\hline \multicolumn{5}{|c|}{$\begin{array}{l}\text { Puntuación (ítems en cada subescala y valencia): Crítica al sistema/creencias justificativas }(+2,-6,-9,-10,-11,-12,-23, \\
-25) \text {, Libertad/coerción }(+3,-4,+13,+20,-21) \text {, Empoderamiento/paternalismo }(-1,-5,-7,-16,-17,-19,-22,-24) \text {, } \\
\text { Tolerancia/discriminación }(+8,+14,-15,+18) \text {. Antes de realizar cualquier cálculo los ítems negativos deben ser recodificados } \\
\text { si se desean obtener puntuaciones que indiquen respeto por los derechos (y viceversa para vulneración). Recomendamos } \\
\text { promediar los ítems en cada subescala para que todas las puntuaciones tengan un rango de } 1-4 \text {. }\end{array}$} \\
\hline
\end{tabular}


Table 5. English version of the Beliefs and Attitudes towards Mental Health Service Users' Rights Scale including scoring details.

\begin{tabular}{|c|c|c|c|c|}
\hline $\begin{array}{l}\text { In relation to patients with mental health problems and as a } \\
\text { professional I think that... }\end{array}$ & $\begin{array}{l}1 \text { Totally } \\
\text { disagree }\end{array}$ & $\begin{array}{l}2 \\
\text { disagree }\end{array}$ & 3 Agree & $\begin{array}{l}4 \text { Totally } \\
\text { agree }\end{array}$ \\
\hline $\begin{array}{l}\text { The possibility of people with severe mental } \\
\text { disorders having children should be regulated. }\end{array}$ & $\square$ & $\square$ & $\square$ & $\square$ \\
\hline $\begin{array}{l}\text { 2. It is possible to recover without professional } \\
\text { interventions. }\end{array}$ & $\square$ & $\square$ & $\square$ & $\square$ \\
\hline $\begin{array}{l}\text { 3. People should not be involuntarily hospitalised if } \\
\text { they do not pose a threat to the integrity of others }\end{array}$ & $\square$ & $\square$ & $\square$ & $\square$ \\
\hline $\begin{array}{l}\text { 4. Sometimes it is necessary to mechanically restrain } \\
\text { patients. }\end{array}$ & $\square$ & $\square$ & $\square$ & $\square$ \\
\hline $\begin{array}{l}\text { 5. Patients with severe mental disorders require clearer } \\
\text { instructions than other patients. }\end{array}$ & $\square$ & $\square$ & $\square$ & $\square$ \\
\hline 6. Mental disorders are diseases like any other. & $\square$ & $\square$ & $\square$ & $\square$ \\
\hline $\begin{array}{l}\text { 7. Professionals should have more say than patients in } \\
\text { making treatment decisions. }\end{array}$ & $\square$ & $\square$ & $\square$ & $\square$ \\
\hline $\begin{array}{l}\text { 8. Individuals incapacitated by severe mental health } \\
\text { problems should have the right to vote. }\end{array}$ & $\square$ & $\square$ & $\square$ & $\square$ \\
\hline $\begin{array}{l}\text { 9. When patients behave aggressively it is due to their } \\
\text { mental disorder. }\end{array}$ & $\square$ & $\square$ & $\square$ & $\square$ \\
\hline $\begin{array}{l}\text { 10. Declaring someone with a severe mental disorder } \\
\text { incapacitated is a good way of taking care of that } \\
\text { person. }\end{array}$ & $\square$ & $\square$ & $\square$ & $\square$ \\
\hline $\begin{array}{l}\text { 11. Individuals with mental disorders currently have the } \\
\text { same rights as other people. }\end{array}$ & $\square$ & $\square$ & $\square$ & $\square$ \\
\hline $\begin{array}{l}\text { 12. Coercive measures are currently applied only when } \\
\text { necessary. }\end{array}$ & $\square$ & $\square$ & $\square$ & $\square$ \\
\hline $\begin{array}{l}\text { 13. When a patient behaves aggressively it is due to the } \\
\text { situations that occur, for example in involuntary } \\
\text { admissions. }\end{array}$ & $\square$ & $\square$ & $\square$ & $\square$ \\
\hline $\begin{array}{l}\text { 14. I would feel comfortable making friends with } \\
\text { someone with a severe mental disorder. }\end{array}$ & $\square$ & $\square$ & $\square$ & $\square$ \\
\hline $\begin{array}{l}\text { 15. I am uncomfortable with patients who regularly use } \\
\text { emergency services. }\end{array}$ & $\square$ & $\square$ & $\square$ & $\square$ \\
\hline $\begin{array}{l}\text { 16. People with severe mental disorders always require } \\
\text { support to be able to live independently. }\end{array}$ & $\square$ & $\square$ & $\square$ & $\square$ \\
\hline $\begin{array}{l}\text { 17. Objective tests should be prioritised over the } \\
\text { professionals' and patients' opinion. }\end{array}$ & $\square$ & $\square$ & $\square$ & $\square$ \\
\hline $\begin{array}{l}\text { 18. I would be comfortable if a person with a mental } \\
\text { disorder were a teacher in a school. }\end{array}$ & $\square$ & $\square$ & $\square$ & 口 \\
\hline $\begin{array}{l}\text { 19. Respecting the patients' dignity is important, but } \\
\text { some aspects of treatment may require flexibility. }\end{array}$ & $\square$ & $\square$ & $\square$ & $\square$ \\
\hline $\begin{array}{l}\text { 20. Greater importance should be placed on promoting } \\
\text { the patient's independence than on reducing the } \\
\text { patient's symptoms. }\end{array}$ & $\square$ & $\square$ & $\square$ & $\square$ \\
\hline $\begin{array}{l}\text { 21. If there are not enough staff, mechanical restraints } \\
\text { are the only way to manage violent situations. }\end{array}$ & $\square$ & $\square$ & $\square$ & $\square$ \\
\hline $\begin{array}{l}\text { 22. When dealing with patients it is important for me } \\
\text { not to get emotionally involved. }\end{array}$ & $\square$ & $\square$ & $\square$ & $\square$ \\
\hline 23. Some patients will never be able to recover. & $\square$ & $\square$ & $\square$ & $\square$ \\
\hline $\begin{array}{l}\text { 24. In my clinical practice I try to leave my personal } \\
\text { values aside. }\end{array}$ & $\square$ & $\square$ & $\square$ & $\square$ \\
\hline $\begin{array}{l}\text { 25. For the most part, mental health professionals work } \\
\text { collaboratively with patients. }\end{array}$ & $\square$ & $\square$ & $\square$ & $\square$ \\
\hline \multicolumn{5}{|c|}{$\begin{array}{l}\text { Scoring (items in each subscale and valence): System criticism/justifying beliefs }(+2,-6,-9,-10,-11, \\
-12,-23,-25) \text {, Freedom/coercion }(+3,-4,+13,+20,-21) \text {, Empowerment/paternalism }(-1,-5,-7,-16, \\
-17,-19,-22,-24) \text {, Tolerance/discrimination }(+8,+14,-15,+18) \text {. Before performing any calculations, } \\
\text { negative items must be recoded if scores indicating respect for rights want to be obtained (and vice versa } \\
\text { for violation). We recommend averaging the items in each subscale so that all scores have a range of } 1-4 \text {. }\end{array}$} \\
\hline
\end{tabular}




\section{Discussion}

According to our results, the BAMHS may be a useful tool to assess the impact of awareness and training activities on professionals' beliefs and attitudes towards service users' rights. This new scale offers flexibility and assumes no prior awareness or knowledge, making it especially suitable for its use in areas where user-led and progressive professional movements are carrying out activities with professionals without previous recovery knowledge or awareness of user rights violations.

The results illustrate four final dimensions, namely: system criticism/justifying beliefs, freedom/coercion, empowerment/paternalism and tolerance/discrimination. The scale can be scored conveniently in any direction, with higher scores signifying higher respect or a higher violation of rights. We simply advise potential users to make it clear in the methodology of their research report. The final structure of the BAMHS showed an adequate fit according to CFA parameters, good reliability, and good discrimination parameters. Adding six items to the core model did not substantively affect the overall fit of the model, nor that of each of the modified dimensions or the discrimination capacity of each of the items. Additionally, none of the items that were included worsened the reliability of each dimension or the whole model.

The first dimension of the BAMHS materialises the professional beliefs that health-related professionals have which justify the status quo. Claiming that mental disorders are diseases like any other, that their aggressiveness is due to their mental disorders, that it is not possible to recover without the intervention of a professional, and even that some patients will never recover are statements that reinforce the need for mental health staff and their interventions. Regarding the former topic, some authors have stressed the role of biological and genetic attributions in the process of stigmatisation, including the belief that most mental disorders are chronic conditions [54,55]. In some way, understanding that mental disorders are unrecoverable biological conditions might tip the moral balance towards the justification of coercion [56]. Accordingly, professionals scoring high on this subscale might also think that they only use these measures when necessary. In this context, declaring someone incapacitated might be considered an adequate way of care. Finding a justification for the use of extraordinary measures in the very nature of mental disorders might facilitate the concealment between the use of such measures and stating that "mental health service users now have the same rights as other people" and that "mental health professionals, in general, work collaboratively with patients". These types of assertions are related to the complacency usually found among some mental health professionals despite the continuous use of coercion [57-59].

The freedom/coercion dimension addresses recurrent topics with mental health professionals when discussing service users' rights. The subscale includes questions on involuntary hospitalization, mechanical restraints, and, inversely, respect for service users' autonomy. We would like to highlight that more than half of our sample believed that mechanical restraints are sometimes necessary and that one should be involuntarily hospitalised even if they do not pose a threat to others. This is in contrast to the evidence that shows a worse prognosis [60], iatrogenesis [61,62], and even death [63] for people subjected to such coercive measures. Conversely, restraint reduction has been shown to be feasible [64] and to reduce the risk of injury and medical leave among nursing staff [65].

The next subscale, empowerment/paternalism, represents a series of beliefs related to the supposed inability of people diagnosed with mental disorders to take charge of their lives including having children, making decisions regarding their treatment, or prioritizing treatment over dignity [66,67]. This justifies paternalism in the form of guidelines and constant support, with emotionally distant and value-free practices [68].

Finally, the fourth subscale tolerance/discrimination materialises widespread prejudices towards mental health service users. Discrimination occurs in different contexts; for instance, this can include employment discrimination (as many would not feel comfortable with a diagnosed teacher) which is evidenced through low occupational rates [69]. Likewise, social distance reflects the main reason for the stigma that people with mental health problems experience [70,71]. Other discriminatory practices include access to healthcare [72], and those included in legislation, such as the prohibition to vote [73]. 
The main limitation of this validation study is the use of a convenience sample formed by professionals willing to participate in awareness activities. This may have caused biases, such as social desirability, due to the profile of the participants in the activities in which this validation is contextualised. However, this scale is designed to evaluate changes in professionals willing to participate in activities where patients' rights are discussed. Therefore, we believe that it can be a useful tool to evaluate awareness activities in the mental health field. Future work should culturally and linguistically adapt the tool for other territories and establish psychometric properties.

We believe that the BAMHS, a relatively brief scale tested in diverse mental health provision contexts with a wide range of professionals, can be used to measure the impact of recovery and anti-stigma Targeted, Local, Credible, Continuous Contact (TLC3) methodology-based interventions [74] carried with mental health professionals.

\section{Conclusions}

In conclusion, we believe that our instrument brings a new perspective to the measurement of beliefs and attitudes of mental health professionals in the context of the new era opened by the Convention on the Rights of Persons with Disabilities [75].

Supplementary Materials: The following are available online at http://www.mdpi.com/1660-4601/16/2/244/s1. Table S1, Descriptive data of the initial 44 item pool; Table S2, Evolution of confirmatory factor analysis fit and discrimination parameters.

Author Contributions: Conceptualization, F.J.E.O.; methodology, F.J.E.O.; software, F.J.E.O.; validation, F.J.E.O.; formal analysis, F.J.E.O.; investigation, F.J.E.O.; resources, F.J.E.O.; data curation, L.L.-B.; writing—original draft preparation, L.L.-B. and F.J.E.O.; writing-review and editing, F.J.E.O.; supervision, F.J.E.O.; project administration, F.J.E.O.; funding acquisition, F.J.E.O.

Funding: Dr. Eiroa-Orosa has received funding from the European Union's Framework Programme for Research and Innovation Horizon 2020 (2014-2020) under Marie Skłodowska-Curie Grant Agreement No 654808.

Acknowledgments: We would like to thank all colleagues of the Veus and Catalonia Mental Health Federations as well as the Catalan Alliance Against Stigma, Obertament, for their inputs, support and constant struggle. We also would like to thank William Bromage, Alicia Georghiades, Tim Lomas, Kirsten MacLean, Michael Rowe and Adil Qureshi for their help with the English version of the instrument.

Conflicts of Interest: The authors declare no conflict of interest.

\section{References}

1. Eiroa-Orosa, F.J.; Rowe, M. Taking the concept of citizenship in mental health across countries. Reflections on transferring principles and practice to different sociocultural contexts. Front. Psychol. 2017, 8, 1020. [CrossRef] [PubMed]

2. Davidson, L. The recovery movement: Implications for mental health care and enabling people to participate fully in life. Health Aff. 2016, 35, 1091-1097. [CrossRef] [PubMed]

3. Henderson, C.; Noblett, J.; Parke, H.; Clement, S.; Caffrey, A.; Gale-Grant, O.; Schulze, B.; Druss, B.; Thornicroft, G. Mental health-related stigma in health care and mental health-care settings. Lancet Psychiatry 2014, 1, 467-482. [CrossRef]

4. Knaak, S.; Mantler, E.; Szeto, A. Mental illness-related stigma in healthcare. Healthc. Manag. Forum 2017, 30, 111-116. [CrossRef] [PubMed]

5. Schomerus, G.; Schwahn, C.; Holzinger, A.; Corrigan, P.W.; Grabe, H.J.; Carta, M.G.; Angermeyer, M.C. Evolution of public attitudes about mental illness: A systematic review and meta-analysis. Acta Psychiatr. Scand. 2012, 125, 440-452. [CrossRef]

6. Hansson, L.; Jormfeldt, H.; Svedberg, P.; Svensson, B. Mental health professionals' attitudes towards people with mental illness: Do they differ from attitudes held by people with mental illness? Int. J. Soc. Psychiatry 2011, 59, 48-54. [CrossRef] [PubMed]

7. Sheehan, L.; Nieweglowski, K.; Corrigan, P.W. Structures and types of Stigma. In The Stigma of Mental Illness-End of the Story? Springer: Cham, Switzerland, 2017; pp. 43-66. 
8. Lorem, G.F.; Hem, M.H.; Molewijk, B. Good coercion: Patients' moral evaluation of coercion in mental health care. Int. J. Ment. Health Nurs. 2015, 24, 231-240. [CrossRef]

9. Mabe, P.A.; Rollock, M.; Duncan, G.N. Teaching clinicians the practice of recovery-oriented care. In Handbook of Recovery in Inpatient Psychiatry; Singh, N.N., Barber, J.W., Van Sant, S., Eds.; Springer: Cham, Switzerland, 2016; pp. 81-97.

10. Ponce, A.N.; Clayton, A.; Gambino, M.; Rowe, M. Social and clinical dimensions of citizenship from the mental health-care provider perspective. Psychiatr. Rehabil. J. 2016, 39, 161-166. [CrossRef]

11. Gilbert, D.C.; Levinson, D.J. Ideology, personality, and institutional policy in the mental hospital. J. Abnorm. Soc. Psychol. 1956, 53, 263-271. [CrossRef]

12. Rogers, A.H.; Cohen, M.; Naranick, C.S. A validation study of the custodial mental illness ideology scale. J. Clin. Psychol. 1958, 14, 269-270. [CrossRef]

13. Cohen, J.; Struening, E.L. Opinions about mental illness in the personnel of two large mental hospitals. J. Abnorm. Soc. Psychol. 1962, 64, 349-360. [CrossRef] [PubMed]

14. Goffman, E.; Guinsberg, L. Stigma: Notes on the Management of Spoiled Identity; Prentice-Hall: New Yok, NY, USA; London, UK; Toronto, ON, Canada, 1963.

15. Corrigan, P.W.; Markowitz, F.E.; Watson, A.; Rowan, D.; Kubiak, M.A. An attribution model of public discrimination towards persons with mental illness. J. Health Soc. Behav. 2003, 44, 162-179. [CrossRef] [PubMed]

16. Taylor, S.M.; Dear, M.J. Scaling community attitudes toward the mentally ill. Schizophr. Bull. 1981, 7, $225-240$. [CrossRef] [PubMed]

17. Evans-Lacko, S.; Rose, D.; Little, K.; Flach, C.; Rhydderch, D.; Henderson, C.; Thornicroft, G. Development and psychometric properties of the Reported and Intended Behaviour Scale (RIBS): A stigma-related behaviour measure. Epidemiol. Psychiatr. Sci. 2011, 20, 263-271. [CrossRef] [PubMed]

18. Penn, D.L.; Guynan, K.; Daily, T.; Spaulding, W.D.; Garbin, C.P.; Sullivan, M. Dispelling the Stigma of Schizophrenia: What Sort of Information Is Best? Schizophr. Bull. 1994, 20, 567-578. [CrossRef] [PubMed]

19. Evans-lacko, S.; Little, K.; Meltzer, H.; Rose, D.; Rhydderch, D.; Henderson, C.; Thornicroft, G. Mental Health Knowledge Schedule. Can. J. Psychiatry 2010, 55, 157-165. [CrossRef] [PubMed]

20. Link, B.G.; Cullen, F.T.; Frank, J.; Wozniak, J.F. The Social Rejection of Former Mental Patients: Understanding Why Labels Matter. Am. J. Sociol. 1987, 92, 1461-1500. [CrossRef]

21. Crisp, A.H.; Gelder, M.G.; Rix, S.; Meltzer, H.I.; Rowlands, O.J. Stigmatisation of people with mental illnesses. Br. J. Psychiatry 2000, 177, 4-7. [CrossRef]

22. Teachman, B.A.; Wilson, J.G.; Komarovskaya, I. Implicit and Explicit Stigma of Mental Illness in Diagnosed and Healthy Samples. J. Soc. Clin. Psychol. 2006, 25, 75-95. [CrossRef]

23. Ritsher, J.B.; Otilingam, P.G.; Grajales, M. Internalized stigma of mental illness: Psychometric properties of a new measure. Psychiatry Res. 2003, 121, 31-49. [CrossRef]

24. Kassam, A.; Papish, A.; Modgill, G.; Patten, S. The development and psychometric properties of a new scale to measure mental illness related stigma by health care providers: The Opening Minds Scale for Health Care Providers (OMS-HC). BMC Psychiatry 2012, 12, 62. [CrossRef]

25. Stull, L.G.; McConnell, H.; McGrew, J.; Salyers, M.P. Explicit and implicit stigma of mental illness as predictors of the recovery attitudes of assertive community treatment practitioners. Isr. J. Psychiatry Relat. Sci. 2017, 54, 31-38. [PubMed]

26. Gabbidon, J.; Clement, S.; van Nieuwenhuizen, A.; Kassam, A.; Brohan, E.; Norman, I.; Thornicroft, G. Mental Illness: Clinicians' Attitudes (MICA) Scale-Psychometric properties of a version for healthcare students and professionals. Psychiatry Res. 2013, 206, 81-87. [CrossRef] [PubMed]

27. Christison, G.W.; Haviland, M.G.; Riggs, M.L. The Medical Condition Regard Scale. Acad. Med. 2002, 77, 257-262. [CrossRef]

28. Esen Danacı, A.; Balıkçı, K.; Aydın, O.; Cengisiz, C.; Uykur, A.B. The Effect of Medical Education On Attitudes Towards Schizophrenia: A Five-Year Follow-Up Study. Turk. J. Psychiatry 2016, 27, 176-184. [CrossRef]

29. Watson, A.C.; Angell, B.; Vidalon, T.; Davis, K. Measuring perceived procedural justice and coercion among persons with mental illness in police encounters: The Police Contact Experience Scale. J. Commun. Psychol. 2010, 38, 206-226. [CrossRef]

30. Burra, P.; Kalin, R.; Leichner, P.; Waldron, J.J.; Handforth, J.R.; Jarrett, F.J.; Amara, I.B. The ATP 30-A scale for measuring medical students' attitudes to psychiatry. Med. Educ. 1982, 16, 31-38. [CrossRef] [PubMed] 
31. Bedregal, L.E.; O'Connell, M.; Davidson, L. The Recovery Knowledge Inventory: Assessment of Mental Health Staff Knowledge and Attitudes about Recovery. Psychiatr. Rehabil. J. 2006, 30, 96-103. [CrossRef]

32. Mabe, P.A.; Fenley, G. Project GREAT Recovery Based Training Procedures Manual. Ph.D Dissertation, Department of Psychiatry and Health Behavior Medical College of Georgia, Augusta, GA, USA, 2008.

33. Salyers, M.P.; Rollins, A.L.; Bond, G.R.; Tsai, J.; Moser, L.; Brunette, M.F. Development of a scale to assess practitioner knowledge in providing integrated dual disorders treatment. Adm. Policy Ment. Health Ment. Health Serv. Res. 2007, 34, 570-581. [CrossRef]

34. Borkin, J.R.; Steffen, J.J.; Ensfield, L.B.; Krzton, K.; Wishnick, H.; Wilder, K.; Yangarber, N. Recovery Attitudes Questionnaire: Development and evaluation. Psychiatr. Rehabil. J. 2000, 24, 95-102. [CrossRef]

35. Salyers, M.P.; Brennan, M.; Kean, J. Provider Expectations for Recovery Scale: Refining a measure of provider attitudes. Psychiatr. Rehabil. J. 2013, 36, 153-159. [CrossRef] [PubMed]

36. Casper, E.S.; Oursler, J.; Schmidt, L.T.; Gill, K.J. Measuring practitioners' beliefs, goals, and practices in psychiatric rehabilitation. Psychiatr. Rehabil. J. 2002, 25, 223-234. [CrossRef] [PubMed]

37. Deane, F.P.; Goff, R.O.; Pullman, J.; Sommer, J.; Lim, P. Changes in Mental Health Providers' Recovery Attitudes and Strengths Model Implementation Following Training and Supervision. Int. J. Ment. Health Addict. 2018. [CrossRef]

38. Killaspy, H.; White, S.; Wright, C.; Taylor, T.L.; Turton, P.; Schützwohl, M.; Schuster, M.; Cervilla, J.A.; Brangier, P.; Raboch, J.; et al. The development of the Quality Indicator for Rehabilitative Care (QuIRC): A measure of best practice for facilities for people with longer term mental health problems. BMC Psychiatry 2011, 11, 35. [CrossRef] [PubMed]

39. Strating, M.M.H.; Broer, T.; van Rooijen, S.; Bal, R.A.; Nieboer, A.P. Quality improvement in long-term mental health: Results from four collaboratives. J. Psychiatr. Ment. Health Nurs. 2012, 19, 379-388. [CrossRef] [PubMed]

40. Drake, C.E.; Codd, R.T.; Terry, C. Assessing the validity of implicit and explicit measures of stigma toward clients with substance use disorders among mental health practitioners. J. Context. Behav. Sci. 2018, 8, 44-54. [CrossRef]

41. Mancini, A.; Finnerty, M. Recovery-Oriented Practices Index (ROPI); University of Minnesota: New York, NY, USA, 2005.

42. O'Connell, M.; Tondora, J.; Croog, G.; Evans, A.; Davidson, L. From Rhetoric to Routine: Assessing Perceptions of Recovery-Oriented Practices in a State Mental Health and Addiction System. Psychiatr. Rehabil. J. 2005, 28, 378-386. [CrossRef]

43. Moors, G. Exploring the effect of a middle response category on response style in attitude measurement. Qual. Quant. 2008, 42, 779-794. [CrossRef]

44. Revelle, W. An Introduction to Psychometric Theory with Applications in R. Available online: https: //personality-project.org/r/book/ (accessed on 14 January 2019).

45. Rosseel, Y. lavaan: An R Package for Structural Equation Modeling. J. Stat. Softw. 2012, 48, 1-21. [CrossRef]

46. Rizopoulos, D. ltm: An R package for latent variable modeling and item response theory analyses. J. Stat. Softw. 2006, 17, 1-25. [CrossRef]

47. Chalmers, R.P. mirt: A Multidimensional Item Response Theory Package for the R Environment. J. Stat. Softw. 2012, 48, 1-29. [CrossRef]

48. R Core Team R: A language and Environment For Statistical Computing. R Foundation for Statistical Computing: Vienna, Austria. Available online: https://www.r-project.org/ (accessed on 14 January 2019).

49. Penn, D.L.; Kommana, S.; Mansfield, M.; Link, B.G. Dispelling the Stigma of Schizophrenia: II. The Impact of Information on Dangerousness. Schizophr. Bull. 1999, 25, 437-446. [CrossRef] [PubMed]

50. Kidd, S.A.; George, L.; O’Connell, M.; Sylvestre, J.; Kirkpatrick, H.; Browne, G.; Odueyungbo, A.O.; Davidson, L. Recovery-oriented service provision and clinical outcomes in assertive community treatment. Psychiatr. Rehabil. J. 2011, 34, 194-201. [CrossRef] [PubMed]

51. Barbic, S.P.; Kidd, S.A.; Davidson, L.; McKenzie, K.; O'Connell, M.J. Validation of the Brief Version of the Recovery Self-Assessment (RSA-B) Using Rasch Measurement Theory. Psychiatr. Rehabil. J. 2015, 38, 349-358. [CrossRef] [PubMed]

52. Salyers, M.P.; Tsai, J.; Stultz, T.A. Measuring recovery orientation in a hospital setting. Psychiatr. Rehabil. J. 2007, 31, 131-137. [CrossRef] [PubMed] 
53. Kassam, A.; Glozier, N.; Leese, M.; Henderson, C.; Thornicroft, G. Development and responsiveness of a scale to measure clinicians' attitudes to people with mental illness (medical student version). Acta Psychiatr. Scand. 2010, 122, 153-161. [CrossRef]

54. Albee, G.W.; Joffe, J.M. Mental Illness Is NOT “an Illness Like Any Other". J. Prim. Prev. 2003, 24, 419-436. [CrossRef]

55. Read, J.; Haslam, N.; Sayce, L.; Davies, E. Prejudice and schizophrenia: A review of the "mental illness is an illness like any other" approach. Acta Psychiatr. Scand. 2006, 114, 303-318. [CrossRef] [PubMed]

56. Molewijk, B.; Kok, A.; Husum, T.; Pedersen, R.; Aasland, O. Staff's normative attitudes towards coercion: The role of moral doubt and professional context-A cross-sectional survey study. BMC Med. Ethics 2017, $18,37$. [CrossRef] [PubMed]

57. Berlin, I.N. Resistance to change in mental health professionals. Am. J. Orthopsychiatry 1969, 39, $109-115$. [CrossRef]

58. Rogers, A.; Pilgrim, D. 'Pulling down churches': Accounting for the British Mental Health Users' Movement. Sociol. Health Ill. 1991, 13, 129-148. [CrossRef]

59. Morley, C. Towards Critical Social Work Practice in Mental Health. J. Progr. Hum. Serv. 2003, 14, 61-84. [CrossRef]

60. Bower, F.L.; McCullough, C.S.; Timmons, M.E. A Synthesis of What We Know About the Use of Physical Restraints and Seclusion with Patients in Psychiatric and Acute Care Settings: 2003 Update. Worldviews Evid.-Based Nurs. 2003, 10, 1-29. [CrossRef]

61. Laursen, S.B.; Jensen, T.N.; Bolwig, T.; Olsen, N.V. Deep venous thrombosis and pulmonary embolism following physical restraint. Acta Psychiatr. Scand. 2005, 111, 324-327. [CrossRef] [PubMed]

62. Nielsen, A.S. Deep venous thrombosis and fatal pulmonary embolism in a physically restrained patient. Ugeskr. Laeger 2005, 167, 2294. [PubMed]

63. Morrison, A.; Sadler, D. Death of a Psychiatric Patient during Physical Restraint. Excited Delirium-A Case Report. Med. Sci. Law 2001, 41, 46-50. [CrossRef] [PubMed]

64. Goulet, M.-H.; Larue, C.; Dumais, A. Evaluation of seclusion and restraint reduction programs in mental health: A systematic review. Aggress. Violent Behav. 2017, 31, 413-424. [CrossRef]

65. Lebel, J.; Goldstein, R. The economic cost of using restraint and the value added by restraint reduction or elimination. Psychiatr. Serv. 2005, 56, 1109-1114. [CrossRef]

66. Breeze, J. Can paternalism be justified in mental health care? J. Adv. Nurs. 1998, 28, 260-265. [CrossRef] [PubMed]

67. Pelto-Piri, V.; Engström, K.; Engström, I. Paternalism, autonomy and reciprocity: Ethical perspectives in encounters with patients in psychiatric in-patient care. BMC Med. Ethics 2013, 14, 49. [CrossRef]

68. Sisti, D.; Young, M.; Caplan, A. Defining mental illnesses: Can values and objectivity get along? BMC Psychiatry 2013, 13, 346. [CrossRef] [PubMed]

69. Stuart, H. Mental illness and employment discrimination. Curr. Opin. Psychiatry 2006, 19, 522-526. [CrossRef] [PubMed]

70. Overton, S.L.; Medina, S.L. The Stigma of Mental Illness. J. Couns. Dev. 2008, 86, 143-151. [CrossRef]

71. Singh, N.N.; Barber, J.W.; Van Sant, S. Handbook of Recovery in Inpatient Psychiatry; Singh, N.N., Barber, J.W., Van Sant, S., Eds.; Evidence-Based Practices in Behavioral Health; Springer: Cham, Switzerland, 2016; ISBN 978-3-319-40535-3.

72. Thornicroft, G.; Rose, D.; Kassam, A.; Kassman, A. Discrimination in health care against people with mental illness. Int. Rev. Psychiatry 2007, 19, 113-122. [CrossRef] [PubMed]

73. Lawn, S.; McMillan, J.; Comley, Z.; Smith, A.; Brayley, J. Mental health recovery and voting: Why being treated as a citizen matters and how we can do it. J. Psychiatr. Ment. Health Nurs. 2014, 21, 289-295. [CrossRef] [PubMed]

74. Corrigan, P.W. Best Practices: Strategic Stigma Change (SSC): Five Principles for Social Marketing Campaigns to Reduce Stigma. Psychiatr. Serv. 2011, 62, 824-826. [CrossRef] [PubMed]

75. United Nations. Convention on the Rights of Persons with Disabilities. Treaty Ser. 2006, 2515, 3.

(C) 2019 by the authors. Licensee MDPI, Basel, Switzerland. This article is an open access article distributed under the terms and conditions of the Creative Commons Attribution (CC BY) license (http://creativecommons.org/licenses/by/4.0/). 